\title{
NS5 Duals in Supergravity and Double Field Theory
}

\author{
Valentí Vall Camell* \\ Arnold Sommerfeld Center for Theoretical Physics, \\ Theresienstraße 37, 80333 München, Germany \\ Max-Planck-Institut für Physik, \\ Föhringer Ring 6, 80805 München, Germany \\ E-mail: V.Vallephysik.uni-muenchen.de
}

\begin{abstract}
We investigate corrections to the backgrounds obtained by dualising the NS5-brane solution with compact transverse directions. We study the physics of winding modes and argue that some of the effects are missed in the Supergravity description. We discuss how and to which extent these effects are captured by Double Field Theory.
\end{abstract}

Corfu Summer Institute 2016 "School and Workshops on Elementary Particle Physics and Gravity" 31 August - 23 September, 2016

Corfu, Greece

*Speaker. 


\section{Introduction}

Since its discovery, T-duality [1] has been a very important tool to study string backgrounds. In the most simple setting, it tells that strings propagating on a circle of radius $R$ cannot be distinguished from those propagating on a circle of radius $1 / R$. This can be easily generalised to strings on a d-dimensional torus, where the possible dual backgrounds are related by $O(d, d, \mathbb{Z})$ transformations. Furthermore, it is also possible to dualise more general backgrounds, provided they have a compact isometry. Duality transformations for the general case are known in the literature as Buscher rules [2].

Among other implications, T-duality points out a new direction to enlarge the string landscape beyond usual geometry. One can conceive backgrounds where transition functions between patches are generalised to T-duality transformations. From the point of view of classical geometry, these transformations are not diffeomorphisms, so the background is not even a manifold. This motivates the name of non-geometric backgrounds [3, 4]. However, strings do not see such discontinuities due to the mentioned T-duality. Then, from a stringy point of view such backgrounds are smooth.

In recent years, different models for non-geometric models appeared. An important example are T-folds [5, 6], which are non-trivial flat torus fibrations. The torus is allowed to vary smoothly along the base and it is globally well defined only if at the gluing point between patches a Tduality transformation is used. An important example are 2-torus fibration over a 2-dimensional base $[3,17]$. In this situation, the moduli of the torus degenerate at some point of the base and, after encircling this degeneration, the fiber is glued with a T-duality transformation. Such defects are known in the literature as T-fects [17, 18].

Another important observation from T-duality is that it relates two type of string states which are a priory very different: momentum and winding modes. This motivated the appearance of new frameworks such as Generalised Geometry [7, 8] or Double Field Theory (DFT) [9, 10]. In the first the tangent bundle is formally substituted by the sum of the tangent and the cotangent bundle. In this way, winding and momentum are treated in the same manner. In the second, the dimension of the space is doubled to introduce a set of dual coordinates, whose canonical momentum is winding.

In this proceedings paper we discuss T-duality between backgrounds where isometries are present only in certain regions. In particular, we study a concrete example: backgrounds dual to the NS5 brane configuration. After compactifying its transversal space, compact isometries appear in some limit away from the brane, which motivates the use of Buscher rules to find new backgrounds. In the case where two directions are compactified, the configurations in this limit can be understood as a T-fect. When we move close to the brane, however, this description breaks down and corrections describing the exact configuration need to be taken into account. We will analyse these corrections by considering string trajectories in the limit away from the brane. Some of them will introduce dependence of the background fields on dual coordinates. These will be related with winding modes physics, not captured by Supergravity.

We will structure the paper as follows: In the first part we will describe the NS5 dual backgrounds away from the brane after one and two compactifications. We will study string trajectories on them and argue how they are corrected when we move towards the brane. Since dependence on dual space appears, it is useful to study such corrections in a formalism where these coordinates are naturally included, such as DFT. This will be addressed in the second part of the paper. 


\section{Supergravity configurations and T-duality}

Our starting point is the NS5 brane, a well known magnetic five-brane solution of the supergravity equations of motion. On the transversal $\mathbb{R}^{4}$ the configuration reads

$$
\begin{aligned}
d s^{2} & =h\left(r_{4}\right)\left(d r_{4}^{2}+r_{4}^{2} d \Omega_{3}^{2}\right), \\
e^{\Phi} & =g_{s} h\left(r_{4}\right), \\
H & =d B=\star_{4} d h\left(r_{4}\right),
\end{aligned}
$$

where $\star_{4}$ is the 4-dimensional Hodge dual operator, $r_{4}$ is the radial transversal direction and $d \Omega_{3}^{2}$ the volume form on a 3-dimensional sphere. The function $h\left(r_{4}\right)$ is a solution of the co-dimension four harmonic equation, and can be written as

$$
h\left(r_{4}\right)=1+\frac{\sigma}{r_{4}^{2}},
$$

where $\sigma$ is a constant.

\section{1 $S^{1}$ compactifications}

We next compactify the NS5 brane on a circle of radius $R$ by placing the configuration (2.1) on $\mathbb{R}^{3} \times S^{1}$. The compact coordinate, $\xi$, will be defined up to the identification $\xi \sim \xi+2 \pi R$. This situation is equivalent to an array of branes separated by a distance of $2 \pi R$ along $\xi$. The harmonic function becomes

$$
h\left(r_{3}, \xi\right)=1+\sum_{n \in \mathbb{Z}} \frac{\sigma}{r_{3}^{2}+(\xi-2 \pi n R)^{2}},
$$

where $r_{3}$ is the radial direction along the 3 non-compact transversal directions. The series is actually convergent an can be summed analytically to [22]

$$
h\left(r_{3}, \xi\right)=1+\frac{\sigma}{2 R r_{3}} \frac{\sinh \left(r_{3} / R\right)}{\cosh \left(r_{3} / R\right)-\cos (\xi / R)} .
$$

Due to the periodicity of the coordinate $\xi$ the function can also be written as a Fourier series, where the different modes give the order by order corrections to the smearing limit discussed below as

$$
h\left(r_{3}, \xi\right)=1+\frac{\sigma}{2 R r_{3}}\left(1+\sum_{k \neq 0} e^{-|k| r_{3} / R} e^{i k \xi / R}\right) .
$$

\section{Smearing limit and T-duality}

Far away from the brane $\left(r_{3} \gg R\right)$ all modes in the expansion (2.5) are exponentially suppressed and the harmonic function is well approximated by

$$
h\left(r_{3}\right)=1+\frac{\sigma}{2 R r_{3}} .
$$

In this situation, the B-field can be written as $B=\omega \wedge d \xi$, where $\omega$ is a 1-form satisfying $d \omega=$ $\star_{3} d h\left(r_{3}\right)$ with $\star_{3}$ the 3 -dimensional Hodge dual operator. For the following computations, we will use spherical coordinates on the base and take a gauge where

$$
\omega=\frac{\sigma}{2 R}(1-\cos \theta) d \varphi .
$$


We observe that, in this region, all dependence of the background fields on the compact coordinate disappears and therefore this direction becomes a (compact) isometry. The presence of such symmetry allows to apply Buscher rules along this direction and obtain

$$
\begin{aligned}
d s^{2} & =h\left(r_{3}\right)\left(d r_{3}^{2}+r_{3}^{2} d \theta^{2}+r_{3}^{2} \sin ^{2} \theta d \varphi^{2}\right)+\frac{1}{h\left(r_{3}\right)}(d \xi+\omega)^{2}, \\
B & =\Phi=0 .
\end{aligned}
$$

This geometric configuration is an euclidean Taub-NUT space, known in the context of string theory as KK-monopole. Locally it is a non-trivial $S^{1}$ fibration and the fiber shrinks at the origin. However, it is globally $\mathbb{R}^{4}$, since for constant radius the configuration is a Hopf fibration.

\section{Unwinding trajectories and corrections to the smearing limit}

As we shall argue in this section, the duality described above cannot be the full story. Whereas Buscher's approach to T-duality fits perfectly to describe the situation away from the brane, it breaks down when corrections (2.5) are considered. The discussion presented in this section is due to [22], but we will revisit it here since it will be the starting point for the generalisation to the 2-torus case. For details, we refer to the original reference.

The analysis is based on the fact that, even in the smearing limit region, one can find reminiscences of the physics of the full configuration. An example of this is the existence of string trajectories unwinding the fiber away from the origin ${ }^{1}$. These are consequence of the fact that, at constant radius, the background is globally $S^{3}$, which has $\pi_{1}\left(S^{3}\right)=0$. On the smeared NS5 side, the duals of such trajectories do not preserve the canonical momentum ${ }^{2}$ along the direction $\xi$, though in this region translations along it are Killing vectors.

To understand what happens to the winding and momentum charges in these trajectories, it is useful to perform a dimensional reduction on the compact direction and describe the theories from $\mathbb{R}^{3}$ point of view. From this point of view strings with non-trivial momentum or winding along the reduced dimension are seen as charged states. Studying the unwinding trajectories in this context, one observes that in both cases they produce an inflow of current into the brane, while the total charge inside an sphere at infinity is preserved [22]. This implies that the smeared NS5 and the KK-monopole should carry some sort of momentum and winding charge respectively.

For the NS5 case, this analysis is consistent with our previous knowledge of this background: the smearing limit has to be corrected by considering the correction terms in (2.5) that break the isometry by introducing an explicit dependence on the fiber coordinate. Translations along this direction are not symmetries any more and therefore the corresponding momentum is not a conserved quantity.

\footnotetext{
${ }^{1}$ Strings can also unwind at the origin, where the fiber shrinks. However, we are interested in the far away physics and therefore trajectories through the origin are not considered.

${ }^{2}$ In the presence of a B-field, the canonical momentum (which generates translations) is defined as

$$
p_{a}=\int d \sigma\left(g_{a b} \dot{X}^{b}+B_{a b} X^{\prime b}\right) .
$$
}


The KK-monopole case needs a bit more of attention. First, the winding modes couple with the background through the B-field, which is trivial in the configuration (2.8). Second, on the NS5 side the full set of corrections (2.5) localise the brane around some point $\xi_{0} \in S^{1}$ (which we set to $\xi_{0}=0$ for simplicity), which is actually a zero mode of the background. What is the corresponding dual zero mode in the KK-monopole? The answer is in terms of the known harmonic self-dual two-form of the KK-monopole [23],

$$
\Omega=d \Lambda, \quad \Lambda=\frac{R^{2}}{h\left(r_{3}\right)}(d \xi+\omega) .
$$

This allows to construct a B-field of the form

$$
B=\Theta \Omega
$$

where $\Theta$ is a parameter at classical level but promotes to a zero mode when considering a quantum theory. Considering a KK-monopole background with non-zero $\Theta$ and dualising back to the NS5 background, the gauge transformation is mapped into a shift on the fiber coordinate (plus a trivial gauge transformation of the B-field) and furthermore, by looking at the Euclidean world-sheet action on a 2-cycle dual to $\Omega$, the coordinate $\Theta$ can be proved to be periodic with period $2 \pi / R$ [23]. Then, one can identify $\Theta$ as the missing fourth collective coordinate, dual to the position of the NS5 brane along $S^{1}$. A more detailed analysis can be found in [21].

Comparison with the NS5 case suggests that the background should also have corrections similar to (2.5). However, these do not localise the brane in the geometrical space but are of stringy nature. In particular, it is conjectured in [22] that the higher Fourier modes in (2.5) are mapped one by one into stringy corrections of the Taub-NUT space by introducing a dependence on a dual coordinate $\tilde{\xi}$

$$
\mathscr{C}_{k}=e^{-|k| r_{3} / R} e^{i k \xi / R} \stackrel{T \text { duality }}{\longrightarrow} \quad \tilde{\mathscr{C}}_{k}=e^{-|k| r_{3} \tilde{R}} e^{i k \tilde{\xi} \tilde{R}}
$$

where we also introduce the dual radius $\tilde{R}=1 / R$. In the same way that geometrical corrections in the NS5 localise the brane around a point $\xi_{0}$, the dual corrections in the KK-monopole background localise it around the dual collective coordinate $\Theta$. Therefore dual (or winding) coordinates are identified with the dyonic collective coordinate $(2.10)[22,24]$. This conjecture has been checked (to some extend) in [24, 25], where corrections (2.11) are interpreted as worldsheet instanton corrections to T-duality, as it was done on the NS5 side in [20].

\section{2 $T^{2}$ compactifications and T-fects}

Next, we generalise the arguments above and we study the NS5 brane on a transversal space $\mathbb{R}^{2} \times T^{2}$. This is equivalent to an infinite set of branes displayed along a 2-dimensional lattice ${ }^{3}$. Splitting $r_{4}$ into $r_{4}^{2}=r^{2}+\left(\xi^{1}\right)^{2}+\left(\xi^{2}\right)^{2}$, where $\xi^{i}$ are the compact coordinates with periodicity $2 \pi R_{i}$, the background is described by the harmonic function

$$
h\left(r, \xi^{1}, \xi^{2}\right)=1+\sum_{\vec{n} \in \mathbb{Z}^{2}} \frac{\sigma}{r^{2}+\left(\xi^{1}-2 \pi R_{1} n_{1}\right)^{2}+\left(\xi^{2}-2 \pi R_{2} n_{2}\right)^{2}} .
$$

\footnotetext{
${ }^{3}$ For simplicity we consider only rectangular lattices. Generalisation to twisted tori is straightforward.
} 
Unlike the case discussed in the last section, this double sum is not convergent. However, one can perform a regularisation procedure together with a Poisson resummation to obtain [12, 28]

$$
h\left(r, \xi^{1}, \xi^{2}\right)=\frac{\sigma}{2 \pi R_{1} R_{2}}\left[\log \frac{\mu}{r}+\sum_{\vec{k} \in\left(\mathbb{Z}^{2}\right)^{*}} K_{0}(\lambda r) e^{-i\left(\frac{k_{1} \xi^{1}}{R_{1}}+\frac{k_{2} \xi^{2}}{R_{2}}\right)}\right],
$$

where $\left(\mathbb{Z}^{2}\right)^{*}=\mathbb{Z}^{2}-\{(0,0)\}$ and $\mu$ is a parameter that controls the regularisation and absorbs also other possible constants. We also define

$$
\lambda=\sqrt{\left(\frac{k_{1}}{R_{1}}\right)^{2}+\left(\frac{k_{2}}{R_{2}}\right)^{2}} .
$$

\section{Smearing limit, T-fects and T-duality}

Again, it is interesting to study the region where $r \gg R_{1}, R_{2}$. In this limit, the harmonic function (2.13) reduces to

$$
h(r)=\frac{\sigma}{2 \pi R_{1} R_{2}} \log \frac{\mu}{r},
$$

and the compact directions $\xi^{1}$ and $\xi^{2}$ become isometries of the background. The configuration is locally a flat torus fibered over a 2-dimensional base. The corresponding B-field is, up to gauge transformations,

$$
B=\frac{\sigma}{2 \pi R_{1} R_{2}} \theta d \xi^{1} \wedge d \xi^{2}
$$

In the smearing limit, the NS5 with two compact transversal directions can be seen as a codimension two defect, which in this context are generally called T-fects [17]. Such degenerations arise in 2-torus fibrations whose moduli are allowed to vary along a 2 dimensional base and they are characterised by its non-trivial monodromy around them.

More concretely, the moduli space of a 2-dimensional torus can be parametrised by two complex parameters: its complex structure $\tau$ and the Kähler form $\rho$. We use the conventions

$$
\tau=\frac{g_{12}}{g_{22}}+i \frac{\sqrt{\operatorname{det} g}}{g_{22}}, \quad \rho=B+i \sqrt{\operatorname{det} g}
$$

where $g_{a b}$ and $B$ are the components of the metric and the B-field tensors on the torus. In this language, the T-duality group in 2 dimensions factorises into $O(2,2, \mathbb{Z})=S L(2, \mathbb{Z})_{\tau} \times S L(2, \mathbb{Z})_{\rho} \times$ $\mathbb{Z}_{2} \times \mathbb{Z}_{2}$, where the $S L(2, \mathbb{Z})$ factors act on $\tau$ and $\rho$ as Möbius transformations. The first one is actually the group of large diffeomorphisms of the torus, and therefore is usually called the geometric subgroup. The second includes gauge transformations for the B-field and non-geometric T-duality transformations. Finally, the $\mathbb{Z}_{2}$ factors correspond to $\tau \leftrightarrow \rho$ and $(\tau, \rho) \rightarrow(-\bar{\tau},-\bar{\rho})$. The first of this elements corresponds to the factorised duality (Buscher rules) along the direction $\xi^{2}$. The other factorised duality, along $\xi^{1}$, corresponds to $\tau \leftrightarrow-1 / \rho$.

A 2-torus fibered over a 2-dimensional base is a solution of the string background equations of motion if the moduli parameters $\rho$ and $\tau$ are meromorphic functions of the base. It is then possible to construct a class of solutions where the base has a degeneration point idenified by a monodromy in the T-duality group, including also elements in the non-geometric subgroup. A classification of such possible degenerations can be found in $[18,19]$. We now use this formalism to describe the smeared NS5 and its dual backgrounds. 


\begin{tabular}{|c|c|l|}
\cline { 2 - 3 } \multicolumn{1}{c|}{} & Monodromy & Winding/momentum numbers \\
\hline NS5 & $\rho \rightarrow \rho+1$ & $\begin{array}{l}\left(n_{1}, n_{2}\right) \rightarrow\left(n_{1}, n_{2}\right) \\
\left(m_{1}, m_{2}\right) \rightarrow\left(m_{1}+n_{2}, m_{2}-n_{1}\right)\end{array}$ \\
\hline KK monopole & $\tau \rightarrow \tau+1$ & $\begin{array}{l}\left(n_{1}, n_{2}\right) \rightarrow\left(n_{1}, n_{2}-n_{1}\right) \\
\left(m_{1}, m_{2}\right) \rightarrow\left(m_{1}+m_{2}, m_{2}\right)\end{array}$ \\
\hline Q brane & $-\frac{1}{\rho} \rightarrow-\frac{1}{\rho}+1$ & $\begin{array}{l}\left(n_{1}, n_{2}\right) \rightarrow\left(n_{1}+m_{2}, n_{2}-m_{1}\right) \\
\left(m_{1}, m_{2}\right) \rightarrow\left(m_{1}, m_{2}\right)\end{array}$ \\
\hline
\end{tabular}

Table 1: Mondromies of the NS5 dual in the semiflat approximation and its duals. The second column shows how the winding numbers $\left(n_{1}, n_{2}\right)$ and the momentum numbers $\left(m_{1}, m_{2}\right)$ change.

- Smeared NS5. The smeared NS5 brane described by the harmonic function (2.15) is identified with the monodromy $\rho \rightarrow \rho+1$. In this case, after going around the origin, the B-field differs by a total derivative. The background is then globally well defined in terms of classical geometry.

- Smeared KK monopole. Applying Buscher rules to one of the compact isometries of the NS5 brane, one obtains a smeared version of the KK monopole configuration (2.8). In terms of monodromies, this background is described by $\tau \rightarrow \tau+1$. Since the monodromy belongs to the geometric subgroup, the background is a differentiable manifold. In terms of Kodaira classification for degenerations of torus fibrations [16], this corresponds to a $I_{1}$ degeneration.

- Q-brane. Finally, one can dualise the NS5 along both isometries simultaneously ${ }^{4}$ obtaining $[13,14]$

$$
\begin{aligned}
d s^{2} & =h(r)\left(d r^{2}+r^{2} d \theta^{2}\right)+\frac{4 \pi^{2} h(r)}{4 \pi^{2} h(r)^{2}+\tilde{R}_{1}^{2} \tilde{R}_{2}^{2} \theta^{2}}\left[\left(d \xi^{1}\right)^{2}+\left(d \xi^{2}\right)^{2}\right], \\
B & =-\frac{2 \pi \tilde{R}_{1} \tilde{R}_{2} \theta}{4 \pi^{2} h(r)^{2}+\tilde{R}_{1}^{2} \tilde{R}_{2}^{2} \theta^{2}} d \xi^{1} \wedge d \xi^{2}, \\
e^{2 \Phi} & =\frac{4 \pi^{2} h(r)}{4 \pi^{2} h(r)^{2}+\tilde{R}_{1}^{2} \tilde{R}_{2}^{2} \theta^{2}},
\end{aligned}
$$

which has the monodromy $-\frac{1}{\rho} \rightarrow-\frac{1}{\rho}+1$. This configuration is non-geometric and it is globally well defined only if one allows for T-duality transformations as transition functions between patches. In particular, after going $\theta \rightarrow \theta+2 \pi$ the fiber can only be glued together using a transformation that mixes the volume of the torus and the B-field in a non-trivial way.

\section{Unwinding trajectories and corrections}

As we did for the $S^{1}$ case, we will now investigate reminiscence of the physics of the full background by studying unwinding trajectories in the semiflat approximation discussed in the previous section. Describing our backgrounds in terms of their monodromies becomes useful because, after

\footnotetext{
${ }^{4}$ The same configuration can also be obtained stepwise by applying Buscher rules to the smeared KK-monopole configuration. However, stepwise duality has some global issues that are avoided if one considers direct dualisation from the NS5. For a more detailed discussion, see [28].
} 
a small calculation, one can read how momentum and winding numbers change when going around the brane. The cases of our interest are summarised in table 1. Explicit trajectories and their unwinding physics are discussed in [28]. We will here outline the main conclusions and refer to the original reference for details.

- In the NS5 case, only the momentum numbers change around the brane, which allows trajectories along which momentum is not conserved in both toroidal directions after a good choice of initial winding numbers. On the other side, winding numbers are not affected by the monodromy, so strings cannot unwind. The situation is then analogue to the $S^{1}$ case: momentum along both compact directions is not conserved, which is consistent with corrections (2.13), that break both isometries.

- For the smeared KK monopole, we expect corrections in winding space at least in one of the directions. Indeed, the change of winding and momentum numbers while encircling the origin shows that trajectories unwinding $\xi^{2}$ can be constructed. Furthermore, the momentum along this direction is conserved. On the other side, winding along direction $\xi^{1}$ is conserved while momentum is not. This is then consistent with the findings of the $S^{1}$ situation: after compactifying the KK-monopole, corrections in the dual space discussed in (2.11) remain and we get an extra set of geometric corrections that localise the brane along the extra compact direction [11].

- Finally, the analysis can be extended to the Q-brane. In this case, the study of winding and momentum numbers points out that a string winding any direction of the torus can be unwound by a trajectory with an appropriate initial momentum. On the other side, momentum along both coordinates is conserved. This analysis suggests that the Q-brane should have corrections depending on dual coordinates along both directions of the torus.

As we did for the $S^{1}$ case, it is also useful to study the zero modes of such backgrounds though, in this case, the explicit form of the collective coordinates for the Q-brane is not known. However, one can still guess their nature with the help of T-duality. Our starting point is the knowledge that on the smeared NS5 the zero modes are translations along the toroidal coordinates. We consider then the NS5 background and the shifts $\xi^{1} \rightarrow \xi^{1}+f_{1}(r, \theta)$ and $\xi^{2} \rightarrow \xi^{2}+f_{2}(r, \theta)$. Under T-duality along $\xi^{2}, f_{1}$ remains as a coordinate shift of the KK monopole, while $f_{2}$ is mapped to a gauge transformation of the B-field. On the Q-brane configuration, both transformations become gauge transformations and the metric is not affected by the shifts. This fact points in the direction that the Q-brane has two dyonic zero modes, which is consistent with the previous analysis of unwinding trajectories.

\section{Solutions in extended space}

In the previous sections, it was argued the need of corrections depending on dual coordinates in some of the analysed backgrounds. For this reason, it is useful to study such configurations in a formalism that treats on equal footing both geometrical and dual coordinates. Therefore, in this section we will discuss the previous findings using Double Field Theory (DFT) [9, 10]. 


\subsection{Double Field Theory and generalized T-duality}

DFT is defined on a $2 d$-dimensional space with coordinate content $X^{M}=\left(x^{\mu}, \tilde{x}_{\mu}\right)$, where $x^{\mu}$ are the geometrical coordinates and $\tilde{x}_{\mu}$ the corresponding dual ones. The main objects in DFT are the generalised metric, an $O(d, d)$ tensor that encodes the supergravity fields as

$$
\mathscr{H}_{M N}=\left(\begin{array}{cc}
g-B g^{-1} B & B g^{-1} \\
-g^{-1} B & g^{-1}
\end{array}\right)_{M N},
$$

and the generalised dilaton $\tilde{\Phi}$, defined via the relation $e^{-2 \tilde{\Phi}}=\sqrt{\operatorname{det} g} e^{-2 \Phi}$. With them, one can construct an $O(d, d)$-covariant theory described by the action [10]

$$
S \sim \int d^{N} X e^{-2 \tilde{\Phi}} \mathscr{R}(\mathscr{H}, \tilde{\Phi}),
$$

where $\mathscr{R}(\mathscr{H}, \tilde{\Phi})$ is called generalized curvature scalar ${ }^{5}$. If none of the fields depend on the dual coordinates, bosonic Supergravity is recovered after reducing along them.

Within this formalism, factorised duality along direction $x^{a}$ corresponds to the transformation $x^{a} \leftrightarrow \tilde{x}_{a}$. If we consider a supergravity configuration (with no dependence on $\tilde{x}_{a}$ ) which has a compact isometry (no dependence on $x^{a}$ ), the interchange $x^{a} \leftrightarrow \tilde{x}_{a}$ in the doubled space theory is equivalent to apply Buscher rules between the corresponding supergravity configurations. However, since DFT is an $O(d, d)$-covariant theory, if one applies this transformation to a solution which has no isometries, one obtains another DFT solution. This is usually known in the literature as generalised duality [9], and will be used in the next section to discuss dualisation in the localised configurations. In particular, it reproduces the transformation (2.11) for the higher order corrections. Then, the fact that generalised duality applied to the present case gives sensible results might be due to the fact that the directions along which we dualise are compact and a Fourier decomposition of the harmonic function is possible.

\subsection{Localised solutions in Double Field Theory}

With the described generalised duality, one can dualise along the compact coordinates of the localised NS5 described by the harmonic function (2.13). In this situation it is possible to choose a gauge where the B-field for the NS5 is [28]

$$
B=\frac{\theta}{2 \pi R_{1} R_{2}} d \xi^{1} \wedge d \xi^{2}+\Pi_{1} d \theta \wedge d \xi^{1}+\Pi_{2} d \theta \wedge d \xi^{2}
$$

where $\Pi_{1,2}$ are determined by the relation $d B=\star_{4} d h$. Then, applying the duality $\xi^{2} \leftrightarrow \tilde{\xi}_{2}$ one obtains

$$
\begin{aligned}
d s^{2} & =\tilde{h}\left[d r^{2}+r^{2} d \theta^{2}+\left(d \xi^{1}\right)^{2}\right]+\frac{1}{\tilde{h}}\left[d \xi^{2}+\frac{\tilde{R}_{2}}{2 \pi R_{1}} \theta d \xi^{1}+\tilde{\Pi}_{2} d \theta\right]^{2}, \\
B & =\tilde{\Pi}_{1} d \theta \wedge d \xi^{1} \\
e^{2 \Phi} & =\text { const. }
\end{aligned}
$$

\footnotetext{
${ }^{5}$ Despite its name, $\mathscr{H}$ is not a metric tensor for the doubled space and therefore $\mathscr{R}$ does not describe its curvature.
} 
where $\tilde{\Pi}_{1,2} \equiv \Pi_{1,2}\left(r, \xi^{1}, \tilde{\xi}_{2}\right)$ and $\tilde{h} \equiv h\left(r, \xi^{1}, \tilde{\xi}_{2}\right)$, with $h$ the localised harmonic function (2.13), and we write the result in terms of the dual radius $\tilde{R}_{2}=1 / R_{2}$. This background has dependence on the dual coordinate $\tilde{\xi}_{2}$ as expected from our previous analysis. Furthermore, in the limit $r \gg$ $R_{1}, 1 / \tilde{R}_{2}$ the smeared KK-monopole configuration is recovered. A second dualization along the other compact direction, $\xi^{1} \leftrightarrow \tilde{\xi}_{1}$, leads to the background

$$
\begin{aligned}
d s^{2} & =\tilde{h}\left[d r^{2}+r^{2} d \theta^{2}\right]+\frac{4 \pi^{2} \tilde{h}}{4 \pi^{2} \tilde{h}^{2}+\tilde{R}_{1}^{2} \tilde{R}_{2}^{2} \theta^{2}}\left[\left(d \xi^{1}+\tilde{\Pi}_{1} d \theta\right)^{2}+\left(d \xi^{2}+\tilde{\Pi}_{2} d \theta\right)^{2}\right] \\
B & =-\frac{2 \pi \tilde{R}_{1} \tilde{R}_{2} \theta}{4 \pi^{2} \tilde{h}^{2}+\tilde{R}_{1}^{2} \tilde{R}_{2}^{2} \theta^{2}}\left(d \xi^{1}+\tilde{\Pi}_{1} d \theta\right) \wedge\left(d \xi^{2}+\tilde{\Pi}_{2} d \theta\right) \\
e^{2 \Phi} & =\frac{4 \pi^{2} \tilde{h}}{4 \pi^{2} \tilde{h}^{2}+\tilde{R}_{1}^{2} \tilde{R}_{2}^{2} \theta^{2}}
\end{aligned}
$$

where a now $\tilde{\Pi}_{1,2} \equiv \Pi_{1,2}\left(r, \tilde{\xi}_{1}, \tilde{\xi}_{2}\right)$ and $\tilde{h} \equiv h\left(r, \tilde{\xi}_{1}, \tilde{\xi}_{2}\right)$. We also substitute $R_{1,2} \rightarrow \tilde{R}_{1,2}=1 / R_{1,2}$. This background is again consistent with our previous discussion. The Q-brane configuration (2.18) is obtained in the limit $r \gg 1 / \tilde{R}_{1}, 1 / \tilde{R}_{2}$.

Such configurations depend explicitly on the dual coordinates and therefore they are not Supergravity configurations. They have to be understood as DFT solutions by inserting them into (3.1). The corresponding DFT configurations have vanishing generalised curvature $\mathscr{R}_{M N}$ and are therefore solutions to the equations of motion of the action (3.2). A more detailed analysis of such solutions can be found in [28]. Also their near brane limit was considered in [26, 27]

\section{Conclusions}

We investigated T-duality for the NS5-brane solution with compact transverse spaces. For this backgrounds, application of Buscher rules is only possible at a region far away from the brane, where the compact directions become isometries. However, even in this limit one can find reminiscences of the full configuration by analysing string trajectories on them. In particular, winding modes physics was crucial in this analysis. In the dual backgrounds, strings can unwind some of the cycles of the torus without going through the brane. We related this to the fact that such backgrounds get corrections localising them along the dual space.

In the second part, we analysed the localised configurations using generalised T-duality within DFT. The resulting backgrounds are solutions of the DFT equations of motion which depend explicitly on dual coordinates. This results could also be obtained by mapping term by term the localising corrections of the NS5-brane to corrections localising the dual backgrounds along winding space, as in (2.11). This was possible because directions along which dualisation was applied are compact.

Finally, there are still some remaining open questions. T-fects used in the analysis of the $T^{2}$ compactification can be constructed for other monodromies of the T-duality group. Then, analysis of winding and momentum charges can be easily extended to all of them. However, some of this backgrounds are not T-dual to any geometric one, and therefore it is not possible to guess the exact form of the corrections. Furthermore, in general there might be cases where neither momentum nor winding along one direction is preserved. It would then be interesting to analyse the strong constraint for DFT in these situations. 
Acknowledgements We would like to thank D. Lüst, S. Massai and E. Plauschinn for collaboration on related work.

\section{References}

[1] A. Giveon, M. Porrati and E. Rabinovici, "Target space duality in string theory," Phys. Rept. 244 (1994) 77 [hep-th/9401139].

[2] T. H. Buscher, “A Symmetry of the String Background Field Equations," Phys. Lett. B 194 (1987) 59.

[3] S. Hellerman, J. McGreevy and B. Williams, "Geometric constructions of nongeometric string theories," JHEP 0401 (2004) 024 [hep-th/0208174].

[4] S. Hellerman and J. Walcher, "Worldsheet CFTs for Flat Monodrofolds," hep-th/0604191.

[5] C. M. Hull, "A Geometry for non-geometric string backgrounds," JHEP 0510 (2005) 065 [hep-th/0406102].

[6] A. Dabholkar and C. Hull, "Duality twists, orbifolds, and fluxes," JHEP 0309 (2003) 054 [hep-th/0210209].

[7] N. Hitchin, “Generalized Calabi-Yau manifolds,” Quart. J. Math. 54 (2003) 281 [math/0209099 [math-dg]].

[8] M. Gualtieri, "Generalized complex geometry," math/0703298 [math.DG].

[9] C. Hull and B. Zwiebach, "Double Field Theory,” JHEP 0909 (2009) 099 [arXiv:0904.4664 [hep-th]].

[10] O. Hohm, C. Hull and B. Zwiebach, "Generalized metric formulation of double field theory," JHEP 1008 (2010) 008 [arXiv:1006.4823 [hep-th]].

[11] H. Ooguri and C. Vafa, "Summing up D instantons," Phys. Rev. Lett. 77 (1996) 3296 [hep-th/9608079].

[12] K. Becker and S. Sethi, “Torsional Heterotic Geometries,” Nucl. Phys. B 820 (2009) 1 [arXiv:0903.3769 [hep-th]].

[13] J. de Boer and M. Shigemori, "Exotic Branes in String Theory," Phys. Rept. 532 (2013) 65 [arXiv:1209.6056 [hep-th]].

[14] F. Hassler and D. Lust, "Non-commutative/non-associative IIA (IIB) Q- and R-branes and their intersections," JHEP 1307 (2013) 048 [arXiv:1303.1413 [hep-th]].

[15] A. Chatzistavrakidis, F. F. Gautason, G. Moutsopoulos and M. Zagermann, "Effective actions of nongeometric five-branes," Phys. Rev. D 89 (2014) no.6, 066004 [arXiv:1309.2653 [hep-th]].

[16] K. Kodaira, “On compact analytic surfaces i-iii,” Ann. of Math., 71 (1960), 111-152; 77 (1963), 563-626; 78 (1963), 1-40.

[17] D. Lüst, S. Massai and V. Vall Camell, “The monodromy of T-folds and T-fects,” JHEP 1609 (2016) 127 [arXiv:1508.01193 [hep-th]].

[18] A. Font, I. Garcia-Etxebarria, D. Lust, S. Massai and C. Mayrhofer, "Heterotic T-fects, 6D SCFTs, and F-Theory," JHEP 1608 (2016) 175 [arXiv:1603.09361 [hep-th]].

[19] I. García-Etxebarria, D. Lust, S. Massai and C. Mayrhofer, "Ubiquity of non-geometry in heterotic compactifications," JHEP 1703, 046 (2017) [arXiv:1611.10291 [hep-th]]. 
[20] D. Tong, "NS5-branes, T duality and world sheet instantons,” JHEP 0207 (2002) 013 [hep-th/0204186].

[21] E. Witten, “Branes, Instantons, And Taub-NUT Spaces,” JHEP 0906 (2009) 067 [arXiv:0902.0948 [hep-th]].

[22] R. Gregory, J. A. Harvey and G. W. Moore, "Unwinding strings and t duality of Kaluza-Klein and h monopoles,” Adv. Theor. Math. Phys. 1 (1997) 283 [hep-th/9708086].

[23] A. Sen, "Kaluza-Klein dyons in string theory," Phys. Rev. Lett. 79 (1997) 1619 [hep-th/9705212].

[24] J. A. Harvey and S. Jensen, "Worldsheet instanton corrections to the Kaluza-Klein monopole,” JHEP 0510 (2005) 028 [hep-th/0507204].

[25] S. Jensen, “The KK-Monopole/NS5-Brane in Doubled Geometry,” JHEP 1107 (2011) 088 [arXiv:1106.1174 [hep-th]].

[26] D. S. Berman and F. J. Rudolph, “Branes are Waves and Monopoles,” JHEP 1505 (2015) 015 [arXiv:1409.6314 [hep-th]].

[27] I. Bakhmatov, A. Kleinschmidt and E. T. Musaev, "Non-geometric branes are DFT monopoles," JHEP 1610 (2016) 076 [arXiv:1607.05450 [hep-th]].

[28] D. Lüst, S. Massai E. Plauschinn and V. Vall Camell, to appear. 